\title{
Circulatory and Metabolic Effects of Hypoxia in the Hyperinsulinemic Ovine Fetus
}

\author{
BARBARA S. STONESTREET, JOHN A. WIDNESS, AND DENNIS J. BERARD \\ Brown University School of Medicine, Department of Pediatrics, Women and Infants' Hospital of \\ Rhode Island, Providence, Rhode Island 02905 [B. S. S., D.J.B.] and Department of Pediatrics, \\ University of Iowa Hospitals and Clinic Iowa City, Iowa 52242 [J.A.W.]
} \begin{abstract}
ABS
Fetuses of women whose diabetes is poorly controlled often
exhibit hypoxemia and elevated catecholamine concentrations at birth. In the ovine fetus, experimental hyperinsulinemia results in hypoxemia, elevated catecholamine concentrations, and hemodynamic changes. Limited oxygen availability occurring during pregnancy-related complications and/or delivery may present an additional risk to the hyperinsulinemic fetus. In this study, we tested the hypothesis that hypoxia induced via acutely limiting oxygen availability compromises the hemodynamically and metabolically stressed but compensated hyperinsulinemic ovine fetus. Fetuses receiving insulin $(n=8)$ or placebo $(n=5)$ for 48 $\mathrm{h}$ were exposed to maternally induced hypoxia. Hypoxic hypoxia was associated with a surge in catecholamines in the hyperinsulinemic fetuses. During hypoxia, this group exhibited insulinrelated sustained increases in the combined ventricular output and fetal body blood flow, accentuation of the prior insulinrelated increase in blood flow to the heart, decreased systemic
\end{abstract}

Infants born to women with poorly controlled diabetes mellitus have an increased incidence of in utero hypoxemia (1), perinatal asphyxia (2), hypertrophic cardiomyopathy (3, 4), elevated catecholamine concentrations at birth (5), and perinatal mortality, including unexpected sudden fetal death (3). Hyperinsulinemia has been implicated as a major factor in the pathogenesis of many of these morbidities (3). In the ovine fetus, experimental hyperinsulinemia results in hypoxemia (612), elevated catecholamine concentrations $(11,12)$, and hemodynamic changes $(6,9,11,12)$, simulating several of the abnormalities observed in infants of diabetic mothers.

Superimposed hypoxia resulting from abruptio placenta, cord entrapment, and/or difficult or prolonged labor and delivery might present an additional life-threatening risk to the hyperinsulinemic fetus. In the ovine fetus, we (12) and others (6) have previously demonstrated that experimental hyperin-

Received June 8, 1994; accepted February 14, 1995.

Correspondence: Barbara S. Stonestreet, M.D., Women and Infants' Hospital of Rhode Island, 101 Dudley Street, Providence, RI 02905-2499.

Supported by the National Institute of Health and Human Development, Diabetes Center Grant, P50 HD11343. oxygen delivery, accentuation of the insulin-related increased oxygen extraction, reductions in the insulin-related increased systemic oxygen uptake, sustained increases in regional oxygen delivery to the heart and adrenal glands, reductions in the insulinrelated increased delivery to the carcass, and decreased oxygen delivery to the kidneys and gastrointestinal tract. We conclude that, in the hyperinsulinemic ovine fetus, hypoxic hypoxia attenuates the hyperinsulinemia-mediated increased systemic oxygen uptake. Regional oxygen transport is preserved to vital regions (brain, heart, and adrenal glands) by increased perfusion and compromised to certain other regions (kidneys and gastrointestinal tract), because the increases in perfusion are insufficient to offset the limited oxygen availability. (Pediatr Res 38: 67-75, 1995)

$\dot{\mathrm{V}}_{\mathbf{2}}$, oxygen uptake sulinemia results in increased combined ventricular output, and blood flow to the heart, adrenal glands, gastrointestinal tract, kidneys, and carcass. In contrast, experimental fetal hypoxia, without hyperinsulinemia, results in increased blood flow to the fetal brain, heart, and adrenal glands, and in decreased blood flow to the spleen and kidneys $(13,14)$. Experimental hyperinsulinemia and isolated hypoxemia both result in elevations of fetal catecholamine concentrations $(11,12$, 15-17).

Thus, although experimental hyperinsulinemia results in hemodynamic changes with increased perfusion to vital and metabolically active organs (9), superimposed hypoxia may limit oxygen availability to these and other vital fetal tissues. In the present study, we tested the hypothesis that hypoxia induced via acutely limiting fetal oxygen availability compromises the hemodynamically and metabolically stressed but compensated hyperinsulinemic ovine fetus. To study the pathophysiologic effects of hypoxia in the hyperinsulinemic fetus, we measured fetal catecholamine concentrations, circulation, and metabolism during hyperinsulinemia, before and during superimposed maternal hypoxia. 


\section{METHODS}

This study was conducted after approval by the Institutional Animal Care and Use Committee of Brown University and Women and Infants' Hospital of Rhode Island and in accordance with the National Institutes of Health Guidelines for use of experimental animals.

Animal preparation. Surgery was performed on 13 mixed breed pregnant ewes at $120-126 \mathrm{~d}$ of gestation as previously described $(10,12)$. Polyvinyl catheters were placed in the following fetal vessels: inferior vena cava via the saphenous vein for continuous insulin or placebo infusion, the contralateral saphenous vein for radionuclide-labeled microsphere administration and fetal blood replacement, superior vena cava via the jugular vein for radionuclide-labeled microsphere administration, carotid artery for upper body reference sample blood withdrawal and blood sampling, distal aorta via the tibial artery for lower body reference sample withdrawal, the aorta via contralateral tibial artery for continuous blood pressure and heart rate monitoring, and the umbilical vein, via one of its branches in the allantoic sac, for blood sampling. An amniotic fluid catheter was placed for pressure monitoring to correct the fetal arterial and venous pressures. A femoral artery catheter was also placed in the ewe. Catheter care and treatment of the ewes was as previously described $(10,12)$.

Four to nine days after surgery the fetal sheep were assigned to insulin-infused hypoxic $(n=8)$ or placebo-infused hypoxic $(n=5)$ groups. The number of singleton and twin gestations were similar between groups. When a twin gestation was present, one fetus was studied. Studies were performed at 127-133 d of gestation, when the fetal $\mathrm{pH}$ was $\geq 7.30$ and a $\mathrm{PaO}_{2} \geq 16 \mathrm{~mm} \mathrm{Hg}$ at $37^{\circ} \mathrm{C}$.

Experimental protocol. The ewe was brought to the laboratory in a cart $2 \mathrm{~h}$ before the onset of each study. The study consisted of three measurements periods, before fetal infusion (baseline), after $48 \mathrm{~h}$ of insulin or placebo infusion, and during continued infusion and exposure to hypoxia for $1 \mathrm{~h}$, induced by decreasing the maternal inspired oxygen concentration. During each study period, fetal umbilical venous and arterial oxygen content and whole blood glucose concentrations, fetal arterial $\mathrm{pH}$, blood gases, $\mathrm{Hb}$, plasma insulin and catecholamine, and whole blood lactate concentrations and hemodynamic variables including heart rate, mean arterial blood pressure, combined ventricular output, and regional fetal blood flow were measured. Maternal arterial blood samples were also obtained for $\mathrm{pH}$, arterial blood gases, plasma glucose, insulin, and lactate concentrations.

After obtaining the baseline determination, a continuous fetal i.v. infusion of insulin (26 U.day ${ }^{-1}$, Iletin II, Eli Lilly, Indianapolis, IN) or diluent was begun at a rate of $5 \mathrm{~mL} \cdot$ day $^{-1}$, as previously described $(9,11,12)$. After $48 \mathrm{~h}$ of infusion, the measurements were repeated. To achieve equivalent reductions in fetal arterial oxygen content values in both groups, blood gases, and oxygen saturation were periodically measured, and the gas mixture (8-10\% oxygen, $4 \%$ carbon dioxide, and $86-88 \%$ nitrogen) of the ewe was adjusted accordingly. Blood withdrawn for study sampling was replaced with hematocritmatched blood from a donor fetus of similar gestational age. At the conclusion of the period of hypoxia, the ewe and the fetus were then killed (T61 Hoechst-Roussel Agri-Vet, Summerville, NJ) and an autopsy performed to ascertain catheter placement and to procure fetal tissues.

Methodology and analytic methods. Combined ventricular output and regional fetal blood flow were measured as previously described $(12,18)$ using microspheres $15 \mu \mathrm{m}$ in diameter labeled with one of the following radionuclides: ${ }^{46} \mathrm{Sc},{ }^{51} \mathrm{Cr}$, ${ }^{57} \mathrm{Co},{ }^{95} \mathrm{Nb},{ }^{103} \mathrm{Ru}$, or ${ }^{113} \mathrm{Sn}$ (Dupont NEN, Boston, MA). Approximately $1 \times 10^{6}$ of two different radionuclide-labeled microspheres were simultaneously injected into the inferior and superior vena cavae while reference samples of arterial blood were being withdrawn from the carotid artery and lower abdominal aorta. Combined ventricular output and blood flow to the fetal organs were determined on carbonized tissue according to established techniques $(9,12,18)$. Radioactivity of the samples were measured on a $\gamma$ scintillation spectrometer with a Tracor analytic sample changer (model 1185, Elk Grove Village, IL), connected to a Canberra 4203 multichannel pulseheight analyzer (Meridian, CT). Blood flow was calculated using the least-squares method on a MacIntosh computer (Apple Computer, Cupertino, CA) with correction for background counts, isotope decay, and spillover counts as follows:

Blood flow $=\frac{\text { tissue } \mathrm{cpm}}{\text { reference } \mathrm{cpm}}$

$\times$ rate of withdrawal of reference sample

All blood and tissue samples contained sufficient numbers of microspheres to assure accuracy of the blood flow determinations to $\pm 5 \%$ (18). Combined ventricular output was calculated by summation of blood flow to the individual organs and carcass. The carcass represented the remainder of the fetal tissues after the organs had been removed and included mostly skeletal muscle, cartilage, bones, and skin.

Heart rate and mean arterial and amniotic fluid pressures were continuously measured using standard techniques $(9,12)$. Blood gases and $\mathrm{pH}$ were measured on a Corning blood gas analyzer (model 165, Corning Scientific, Medford, MA), Hb concentration and saturation on a Radiometer Hemoximeter (model $\mathrm{OSM}_{2}$, Copenhagen, Denmark), oxygen content in duplicate on a Lex- $\mathrm{O}_{2}$-Con (Lexington Instruments, Waltham, MA), whole blood or plasma glucose in duplicate by the hexokinase method (19), plasma insulin in duplicate by a double-antibody RIA (20), and plasma epinephrine and norepinephrine concentration in duplicate by high pressure liquid chromatography using an electrochemical detection method $(11,21)$. The coefficient of variation between assays for norepinephrine was $9.5 \%$ and for epinephrine $9.4 \%$. The lower limit of sensitivity for this assay was $30 \mathrm{pg}$ for norepinephrine and $15 \mathrm{pg}$ for epinephrine. The recovery of catecholamines from plasma was $80 \%$.

Computations and data analysis. Fetal oxygen delivery, extraction and uptake were calculated with modifications of Fick principle:

Oxygen delivery $=$ placental blood flow

$\times$ umbilical venous oxygen content 
Oxygen uptake $\left(\dot{\mathrm{V}}_{2}\right)=$ placental blood flow

$\times$ umbilical venous-arterial oxygen content

\section{Oxygen extraction ratio $=$ oxygen uptake/delivery}

Similar equations were used for glucose delivery, uptake and extraction. Blood flow to the individual fetal organs were expressed as milliliters $\cdot \min ^{-1} \cdot 100 \mathrm{~g}^{-1}$ wet tissue weight. Oxygen delivery to fetal tissues were calculated using the appropriate upper or lower body oxygen content values. Fetal body and placental and combined ventricular output resistances were calculated by subtracting the appropriate venous pressures from the arterial pressure both corrected for amniotic fluid pressure and divided by the appropriate blood flow.

Analysis of variance for repeated measures was used to examine for significant differences within or between the two study groups. If a significant difference was found within either the insulin- or placebo-infused groups by analysis of variance, Neumann-Keuls post hoc testing was used to detect statistically significant differences among the three measurement periods. When a significant difference was found between the two groups, an unpaired $t$ test with the Bonferroni correction was used to detect differences at specific measurement periods. An $F$ test for interactions was used to detect differences between the two groups over the three study periods. Linear regression analysis was also used to compare blood flow to the heart with the plasma catecholamine concentrations during insulin and placebo infusion with hypoxia, expressed as change from the insulin and placebo infusion values (see Fig. 4). All data were expressed as mean \pm SEM. $p<0.05$ was considered statistically significant.

\section{RESULTS}

The $\mathrm{pH}$, blood gas, oxygen content, $\mathrm{Hb}$, heart rate, and arterial blood pressure values, at baseline, before insulin or placebo infusion, were within the normal range for fetal sheep of this gestational age and were similar between the groups (Table 1). During insulin infusion, arterial oxygen tension and content values decreased, and carbon dioxide tension increased. In the placebo-infused group, changes in these vari- ables were not observed before exposure to hypoxia. During hypoxia in both groups, fetal arterial $\mathrm{pH}$, oxygen tension, and content values decreased, whereas carbon dioxide tension increased. The hypoxia-related decrease in arterial oxygen tension was greater in the placebo- than in the insulin-infused group. Arterial oxygen content values were similar during hypoxia between the groups. The $\mathrm{Hb}$ concentration did not change in either group. After $48 \mathrm{~h}$ of infusion, fetal heart rate was higher in the insulin- than placebo-infused group, and mean arterial blood pressure decreased in the insulin-infused group. Changes were not observed in these variables in the placebo-infused fetal sheep.

The increase in plasma insulin concentration was similar during insulin- and insulin-infusion with hypoxia (Table 2) and did not change in the placebo-infused group. Whole blood glucose concentration decreased in the umbilical vein and fetal artery during insulin and insulin infusion with hypoxia, did not change significantly during placebo infusion and increased during placebo infusion with hypoxia. However, there was an unexplained nonsignificant increase in umbilical venous glucose concentration after $48 \mathrm{~h}$ of placebo infusion. Arterial lactate concentrations did not change significantly during insulin and insulin infusion with hypoxia or placebo infusion, whereas significant increases were observed with hypoxia alone.

Baseline $\mathrm{pH}, 7.47 \pm 0.03, \mathrm{PaO}_{2}, 13.72 \pm 0.76 \mathrm{kPa}, \mathrm{PaCO}_{2}$, $4.56 \pm 0.12 \mathrm{kPa}$, glucose, $2.4 \pm 0.21 \mathrm{mmol} \cdot \mathrm{L}^{-1}$, insulin, $33 \pm$ $15 \mu \mathrm{U} \cdot \mathrm{mL}^{-1}$, lactate, $0.95 \pm 0.09 \mathrm{mmol} \cdot \mathrm{L}^{-1}$, heart rate, 110 \pm 6 beats $\cdot \min ^{-1}$, and mean arterial blood pressure, $90 \pm 4$ $\mathrm{mm} \mathrm{Hg}$ values of the ewes were within the normal range and similar between the groups. The expected hypoxia-related reductions $(p<0.05)$ in arterial oxygen tension in the ewes were also similar between the groups. During hypoxia, the increases in heart rate, $130 \pm 20$ from a baseline of $110 \pm 6$ beats. $\mathrm{min}^{-1}$, and lactate, $1.1 \pm 0.08$ from a baseline of $0.95 \pm$ $0.09 \mathrm{mmol} \cdot \mathrm{L}^{-1}$, in the ewes, whose fetuses received the insulin infusion, were not statistically significant $(p>0.05)$. Significant changes in mean arterial blood pressure, insulin and glucose concentrations from baseline were not observed during the studies in the ewes. Catheter malfunction $(n=2)$ precluded

Table 1. Fetal arterial and hemodynamic values for study groups by study period

\begin{tabular}{|c|c|c|c|c|c|c|}
\hline & \multicolumn{3}{|c|}{ Insulin infusion } & \multicolumn{3}{|c|}{ Placebo infusion } \\
\hline & Baseline & Without hypoxia & With hypoxia & Baseline & Without hypoxia & With hypoxia \\
\hline Time (h) & -1 & 48 & 49 & -1 & 48 & 49 \\
\hline $\mathrm{pH}$ & $7.35 \pm 0.02$ & $7.31 \pm 0.02$ & $7.26 \pm 0.03^{*} \dagger$ & $7.35 \pm 0.01$ & $7.36 \pm 0.02$ & $7.26 \pm 0.02 * \dagger$ \\
\hline $\mathrm{PaO}_{2}(\mathrm{kPa})$ & $2.57 \pm 0.08$ & $2.16 \pm 0.08^{*}$ & $1.72 \pm 0.05^{*} \dagger \dagger \dagger$ & $2.33 \pm 0.05$ & $2.31 \pm 0.11$ & $1.53 \pm 0.02 * \dagger$ \\
\hline $\mathrm{PaCO}_{2}(\mathrm{kPa})$ & $6.56 \pm 0.19$ & $7.20 \pm 0.24^{*}$ & $7.54 \pm 0.25^{*} \dagger$ & $7.09 \pm 0.15$ & $7.01 \pm 0.09$ & $7.70 \pm 0.32 * \dagger$ \\
\hline Base excess $\left(\mathrm{mEq} \cdot \mathrm{L}^{-1}\right)$ & $1.5 \pm 1.1$ & $0.1 \pm 1.3$ & $-2.4 \pm 1.5$ & $2.7 \pm 1.1$ & $3.2 \pm 1.5$ & $-2.1 \pm 1.7$ \\
\hline Oxygen content $\left(\mathrm{mmol} \cdot \mathrm{L}^{-1}\right)$ & $3.14 \pm 0.19$ & $2.53 \pm 0.24^{*}$ & $1.56 \pm 0.25^{*} \dagger$ & $3.10 \pm 0.09$ & $3.13 \pm 0.13$ & $1.64 \pm 0.07 * \dagger$ \\
\hline Hemoglobin $\left(\mathrm{g} \cdot \mathrm{L}^{-1}\right)$ & $100 \pm 3$ & $100 \pm 5$ & $106 \pm 5$ & $106 \pm 3$ & $108 \pm 4$ & $116 \pm 7$ \\
\hline Heart rate (beats $\cdot \min ^{-1}$ ) & $191 \pm 8$ & $227 \pm 10 \dagger \dagger$ & $216 \pm 14$ & $172 \pm 4$ & $161 \pm 16$ & $172 \pm 13$ \\
\hline Mean arterial blood pressure (mm $\mathrm{Hg}$ ) & $46 \pm 2$ & $37 \pm 3^{*}$ & $42 \pm 5$ & $42 \pm 4$ & $43 \pm 5$ & $46 \pm 4$ \\
\hline
\end{tabular}

Values are means $\pm \mathrm{SEM} ; \mathrm{mm} \mathrm{Hg}=\mathrm{kPa} / 0.133$; insulin infused group $n=8$, placebo-infused group $n=5$.

$* p<0.05$ vs baseline within each group.

$\dagger p<0.05 v s$ insulin or placebo infusion (time $=48 \mathrm{~h}$ ) within each group.

$\dagger \dagger p<0.02$ vs placebo-infused group for the same study period by unpaired $t$ test with the Bonferroni correction. 
Table 2. Fetal plasma insulin, whole blood glucose, and lactate concentrations for study groups by study period

\begin{tabular}{|c|c|c|c|c|c|c|}
\hline & \multicolumn{3}{|c|}{ Insulin infusion } & \multicolumn{3}{|c|}{ Placebo infusion } \\
\hline & Baseline & Without hypoxia & With hypoxia & Baseline & Without hypoxia & With hypoxia \\
\hline Time (hours) & -1 & 48 & 49 & -1 & 48 & 49 \\
\hline $\begin{array}{l}\text { Insulin }\left(\mathrm{Ln}, \mu \mathrm{U} \cdot \mathrm{mL}^{-1}\right)^{a} \\
\text { Glucose }\left(\mathrm{mmol} \cdot \mathrm{L}^{-1}\right)\end{array}$ & $3.00 \pm 0.35$ & $5.50 \pm 0.41^{*}$ & $5.49 \pm 0.38^{*}$ & $3.05 \pm 0.37$ & $3.28 \pm 0.22$ & $2.89 \pm 0.25$ \\
\hline Umbilical vein $^{a}$ & $0.85 \pm 0.09(7)$ & $0.56 \pm 0.07^{*} \dagger \dagger(7)$ & $0.58 \pm 0.05 *(7)$ & $0.88 \pm 0.09$ & $1.10 \pm 0.08$ & $1.83 \pm 0.38^{*} \dagger$ \\
\hline Fetal artery ${ }^{a}$ & $0.69 \pm 0.08$ & $0.32 \pm 0.04 *+\dagger$ & $0.37 \pm 0.06^{*}$ & $0.72 \pm 0.08$ & $0.78 \pm 0.08$ & $1.54 \pm 0.33 * \dagger$ \\
\hline Lactate $\left(\mathrm{mmol} \cdot \mathrm{L}^{-1}\right)$ & & & & & & \\
\hline Fetal artery ${ }^{a}$ & $1.18 \pm 0.08$ & $1.25 \pm 0.12$ & $1.62 \pm 0.12$ & $1.10 \pm 0.12$ & $1.25 \pm 0.13$ & $2.65 \pm 0.33^{*} \dagger$ \\
\hline
\end{tabular}

Values are mean $\pm \mathrm{SEM}$; insulin-infused group $n=8$, placebo-infused group $n=5$, unless otherwise indicated in parenthesis; Ln $=$ natural logarithm.

${ }^{a} p<0.05$ refers to designated variables over time by $F$ test for interactions $v s$ placebo-infused group, baseline values were similar between groups.

${ }^{*} p<0.05$ vs baseline within each group.

$\dagger p<0.05$ vs insulin or placebo-infusion (time $=48 \mathrm{~h}$ ) within each group.

$\dagger \dagger p<0.02$ vs placebo-infused group for the same study period by unpaired $t$ test with the Bonferroni correction.

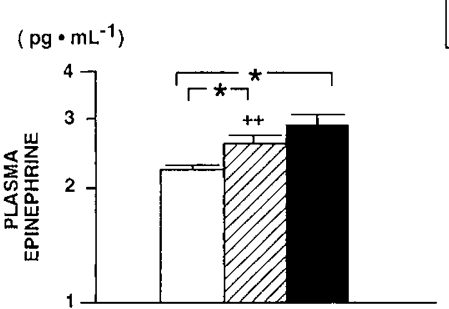

$\square$ Baseline, $\triangle$ Placebo or insulin Infusion

Placebo or Insulin Infusion + Hypoxia.
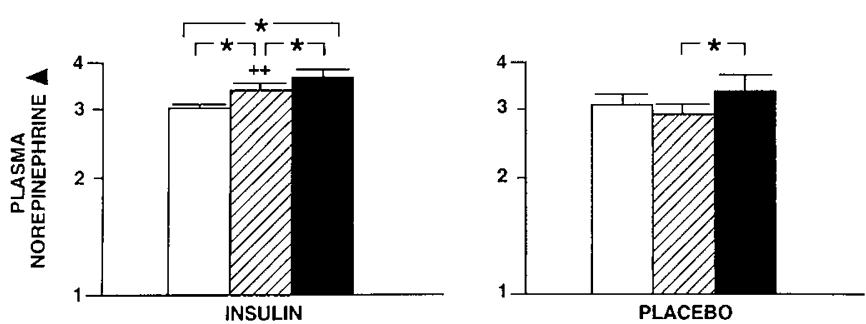

Figure 1. Fetal arterial plasma epinephrine and norepinephrine concentrations ( $\mathrm{Ln}=$ natural logarithm) in the insulin- $(n=8)$ and placebo-infused $(n=5)$ groups. Open bars designate baseline values, before insulin or placebo infusion; hatched bars designate values during insulin or placebo infusion; and solid bars designate values during insulin or placebo infusion with hypoxia. * $p$ $<0.05$ between values indicated by brackets for within group comparisons; $++p<0.05 v s$ placebo-infused group for same study period, $\mathbf{\Delta} p<0.05$ refers to the ordinate axis over time by $F$ test for interactions $v s$ placeboinfused group, baseline epinephrine, and norepinephrine values were similar between the groups.

accurate statistical analysis in the ewes, whose fetuses received the placebo infusion.

After $48 \mathrm{~h}$ of insulin and insulin infusion with hypoxia, epinephrine and norepinephrine concentrations increased significantly (Fig. 1). During insulin infusion, epinephrine and norepinephrine concentrations were significantly higher than during placebo infusion. The increase in norepinephrine was greater during insulin infusion with hypoxia than with infusion alone. In the placebo-infused group, norepinephrine concentrations increased significantly during hypoxia. The pattern of norepinephrine response in the insulin-infused differed significantly from that of the placebo-infused group during the study periods. Baseline epinephrine and norepinephrine concentrations were similar between the insulin- and placebo-infused groups. Although norepinephrine concentration was higher in the insulin-infused compared with the placebo-infused group

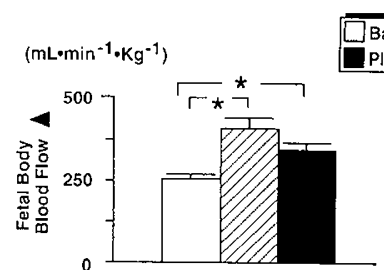

Baseline, $\triangle$ Placebo or insulin infusion, Placebo or Insulin Infusion + Hypoxia.
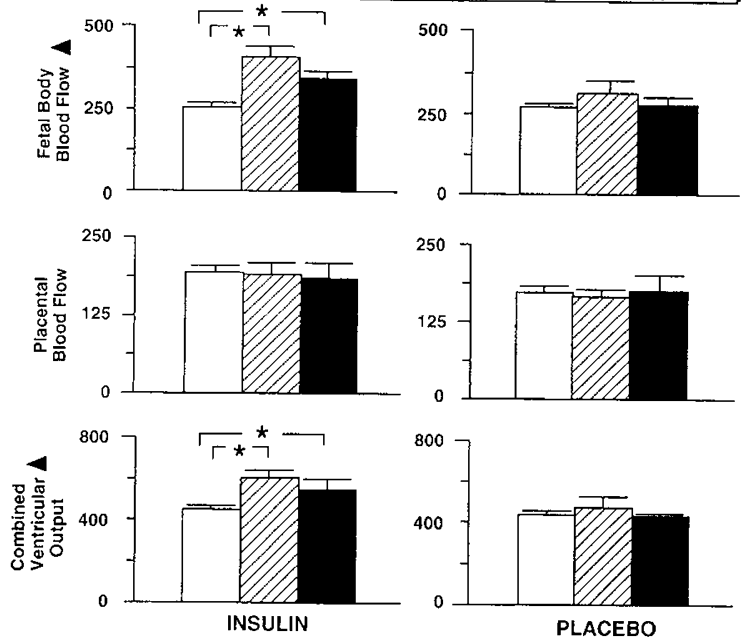

Figure 2. Fetal body blood flow, placental blood flow, and combined ventricular output $\mathrm{mL} \cdot \mathrm{mm}^{-1} \cdot \mathrm{kg}^{-1}$ in the insulin- $(n=8)$ and placebo-infused $(n=5)$ groups. Bar and symbol legends as in Figure 1.

during hypoxia, these differences did not reach statistical significance $(p=0.08)$.

Fetal body blood flow and combined ventricular output increased significantly after $48 \mathrm{~h}$ of insulin infusion and remained increased, when hypoxia was added (Fig. 2). These patterns of fetal body blood flow and combined ventricular output responses in the insulin-infused differed significantly from the placebo-infused group, which did not change during the study periods. Baseline fetal body blood flow and combined ventricular output were similar between the insulin- and placebo-infused groups. Changes in placental blood flow were not observed in either group during any of the three measurement periods. Resistances of the fetal body and combined ventricular output decreased during insulin and insulin infusion with hypoxia (Fig. 3). This pattern of the resistance of the fetal body and combined ventricular output in the insulin-infused differed significantly from that of the placebo-infused group, which did not change during the study periods. Baseline fetal body and combined ventricular output resistances were similar between the insulin- and placebo-infused groups. Placental resistance did not change in either group. 


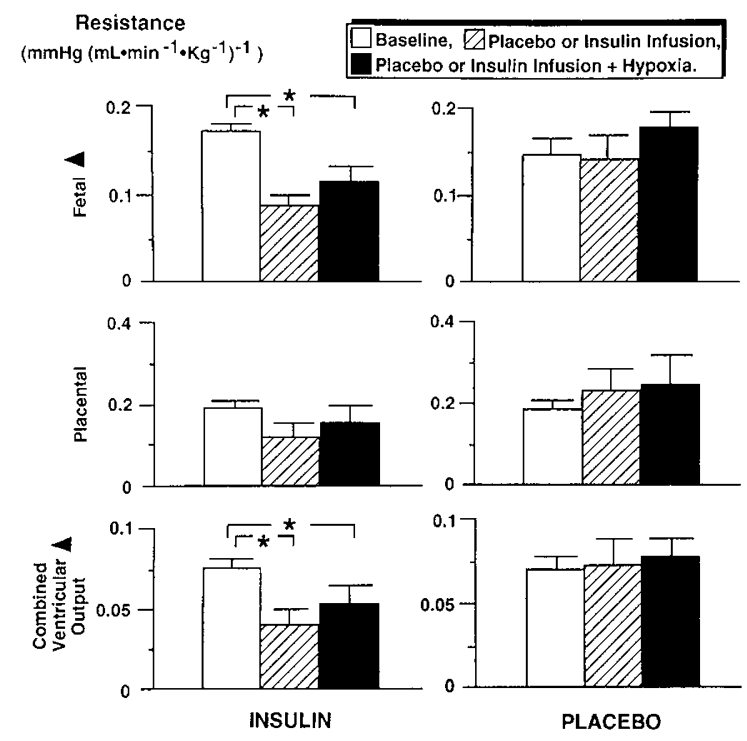

Figure 3. Resistance of the fetal body, placenta, and combined ventricular output in the the insulin- $(n=8)$ and placebo-infused $(n=5)$ groups. Bar and symbol legends as in Figure 1.

Changes in regional fetal blood flow and vascular resistances during insulin infusion and infusion with hypoxia added were noted for some, but not all organs (Table 3). Brain perfusion increased significantly in both groups only during hypoxia. Blood flow to the heart increased 2.3-fold after $48 \mathrm{~h}$ of insulin infusion and 4-fold after insulin infusion with hypoxia. This 4-fold increase was $67 \%$ greater than the 2.4-fold increase over baseline observed in the placebo-infused group during hypoxia $(p<0.02)$. Blood flow to the adrenal glands and liver (hepatic artery) increased significantly compared with baseline values during insulin infusion with hypoxia added. Superimposed hypoxia did not change the prior insulin infusion-related increases in perfusion to the gastrointestinal tract, pancreas, perirenal fat, kidneys, and carcass. Changes in blood flow to the spleen were not observed during insulin infusion or infusion with hypoxia. Regional fetal perfusion was similar to baseline after $48 \mathrm{~h}$ of placebo infusion. When hypoxia was added to placebo infusion, perfusion increased to brain, heart, and adrenal glands and did not change to the other organs. Vascular resistance of the brain decreased compared with baseline values during insulin and insulin infusion with hypoxia added. The decrease in the latter was greater than with infusion alone. Vascular resistance of the heart, adrenal glands, liver, gastrointestinal tract, pancreas, and perinatal fat decreased compared with baseline values during insulin and insulin infusion with hypoxia added. Resistance of the carcass decreased during insulin infusion and returned to values similar to baseline, when hypoxia was added. In the placebo-infused group, vascular resistance decreased to heart and adrenal glands during hypoxia and did not change to other regions.

Blood flow to the heart (Fig. 4) demonstrated a positive $(p<0.05)$ correlation $(r=0.78, n=8)$ with plasma epinephrine concentration during insulin and not placebo infusion $(r=$ $0.48, n=5, p=\mathrm{NS}$ ) with hypoxia, when expressed as change from the insulin or placebo infusion values. Blood flow to the heart did not show a significant correlation with plasma nor-

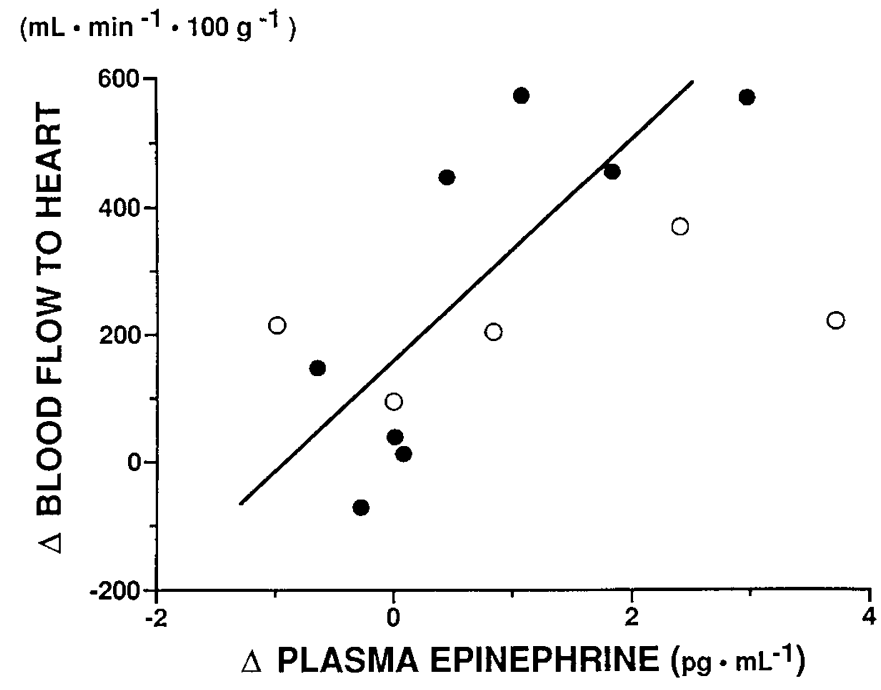

Figure 4. Heart blood flow plotted against plasma epinephrine concentration during insulin (closed circles, $r=0.79, n=8, p<0.05$ ) and placebo infusion (open circles, $r=0.48, n=5, p=\mathrm{NS}$ ) with hypoxia, expressed as change from insulin or placebo infusion values.

epinephrine concentration during insulin infusion $(r=0.49$, $n=8, p=\mathrm{NS})$ or placebo-infusion $(r=0.13, n=5, p=\mathrm{NS})$ with hypoxia, when expressed as change from the insulin or placebo infusion values.

Oxygen delivery to the fetus decreased during hypoxia compared with $48 \mathrm{~h}$ of insulin infusion and baseline values (Fig. 5). Insulin infusion was associated with a significant increase in fetal oxygen extraction, which was augmented by hypoxia. Fetal oxygen uptake increased during insulin infusion and decreased when hypoxia was added to the values, which were similar to baseline. Placebo infusion was not associated with changes in fetal oxygen metabolism. Oxygen delivery to the fetus was reduced, whereas extraction and uptake did not change during hypoxia.

Oxygen delivery to the brain did not change during insulin or insulin infusion with hypoxia (Table 4). The prior insulin infusion-related increases in oxygen delivery to the heart and adrenal glands were sustained during hypoxia. Oxygen delivery to the liver did not change during insulin or insulin infusion with hypoxia, to the gastrointestinal tract decreased during insulin infusion with hypoxia, to the pancreas did not change during insulin or insulin infusion with hypoxia, to the kidneys decreased during insulin infusion with hypoxia compared with infusion alone, to the carcass increased during insulin and was reduced during insulin infusion with hypoxia, and to the spleen did not change during insulin or insulin infusion with hypoxia. Changes in oxygen delivery were not observed after $48 \mathrm{~h}$ of placebo infusion. During hypoxia alone, oxygen delivery increased to the heart and decreased to carcass, gastrointestinal tract, and kidneys. The pattern of the oxygen delivery of the heart and adrenal glands in the insulin-infused group differed significantly over the study periods from that of the placeboinfused group. Baseline values were similar between the groups.

Glucose delivery decreased significantly $(p<0.05)$ from a baseline value of $156 \pm 19 \mu \mathrm{mol} \cdot \mathrm{min}^{-1} \cdot \mathrm{kg}^{-1}$, during insulin 
Table 3. Regional fetal blood flow and vascular resistance for study groups by study period

\begin{tabular}{|c|c|c|c|c|c|c|}
\hline & \multicolumn{3}{|c|}{ Insulin infusion } & \multicolumn{3}{|c|}{ Placebo infusion } \\
\hline & Baseline & Without hypoxia & With hypoxia & Baseline & Without hypoxia & With hypoxia \\
\hline Time (h) & -1 & 48 & 49 & -1 & 48 & 49 \\
\hline \multicolumn{7}{|c|}{ Blood flow $\left(\mathrm{mL} \cdot \mathrm{min}^{-1} \cdot 100 \mathrm{~g}^{-1}\right)$} \\
\hline Brain & $104 \pm 10$ & $128 \pm 14$ & $206 \pm 25^{*} \dagger$ & $121 \pm 8$ & $127 \pm 16$ & $203 \pm 28 * \dagger$ \\
\hline Heart" & $161 \pm 18$ & $372 \pm 35^{*}$ & $647 \pm 107^{*} \dagger \dagger \dagger$ & $162 \pm 9$ & $173 \pm 17$ & $395 \pm 53 * \dagger$ \\
\hline Adrenal glands & $175 \pm 30$ & $552 \pm 95$ & $955 \pm 278^{*}$ & $255 \pm 28$ & $228 \pm 21$ & $737 \pm 133^{*} \dagger$ \\
\hline Liver (hepatic artery) & $5 \pm 2$ & $16 \pm 5$ & $23 \pm 6^{*}$ & $10 \pm 4$ & $14 \pm 3$ & $30 \pm 13$ \\
\hline Gastrointestinal Tract & $61 \pm 9$ & $99 \pm 12^{*}$ & $84 \pm 11 *$ & $68 \pm 6$ & $71 \pm 9$ & $68 \pm 19$ \\
\hline Pancreas & $45 \pm 6$ & $71 \pm 8^{*}$ & $79 \pm 9^{*}$ & $71 \pm 6$ & $83 \pm 18$ & $91 \pm 15$ \\
\hline Perirenal fat ${ }^{a}$ & $28 \pm 5$ & $58 \pm 9^{*}$ & $63 \pm 8^{*}$ & $38 \pm 7$ & $39 \pm 7$ & $40 \pm 6$ \\
\hline Kidneys & $133 \pm 13$ & $219 \pm 29^{*}$ & $193 \pm 29^{*}$ & $156 \pm 9$ & $169 \pm 18$ & $177 \pm 24$ \\
\hline Carcass & $18 \pm 1$ & $31 \pm 2^{*}$ & $27 \pm 3^{*}$ & $22 \pm 1$ & $23 \pm 4$ & $18 \pm 4$ \\
\hline Spleen & $308 \pm 58$ & $418 \pm 126$ & $280 \pm 82$ & $201 \pm 20$ & $370 \pm 50$ & $426 \pm 113$ \\
\hline \multicolumn{7}{|c|}{ Resistance $\left(\mathrm{mmHg}\left(\mathrm{mL} \cdot \mathrm{min}^{-1} \cdot 100 \mathrm{~g}^{-1}\right)^{-1}\right)$} \\
\hline Brain & $0.47 \pm 0.05$ & $0.33 \pm 0.06^{*}$ & $0.22 \pm 0.04 * \dagger$ & $0.35 \pm 0.03$ & $0.37 \pm 0.07$ & $0.25 \pm 0.05$ \\
\hline Heart $^{a}$ & $0.31 \pm 0.03$ & $0.11 \pm 0.01^{*}$ & $0.07 \pm 0.01^{*}$ & $0.27 \pm 0.04$ & $0.27 \pm 0.05$ & $0.13 \pm 0.02 * \dagger$ \\
\hline Adrenal glands ${ }^{a}$ & $0.31 \pm 0.04$ & $0.09 \pm 0.03^{*}$ & $0.07 \pm 0.02 *$ & $0.17 \pm 0.03$ & $0.20 \pm 0.03$ & $0.07 \pm 0.01 * \dagger$ \\
\hline Liver (hepatic artery) & $11.50 \pm 4.34$ & $4.74 \pm 1.58^{*}$ & $4.80 \pm 2.45^{*}$ & $8.39 \pm 2.79$ & $4.26 \pm 1.48$ & $3.26 \pm 1.57$ \\
\hline Gastrointestinal tract ${ }^{a}$ & $0.89 \pm 0.14$ & $0.42 \pm 0.07^{*}$ & $0.59 \pm 0.14 *$ & $0.62 \pm 0.05$ & $0.65 \pm 0.14$ & $0.86 \pm 0.18$ \\
\hline Pancreas $^{a}$ & $1.14 \pm 0.14 \dagger \dagger$ & $0.55 \pm 0.06^{*}$ & $0.60 \pm 0.10^{*}$ & $0.62 \pm 0.11$ & $0.67 \pm 0.20$ & $0.59 \pm 0.10$ \\
\hline Perirenal fat ${ }^{a}$ & $2.12 \pm 0.44$ & $0.73 \pm 0.11^{*}$ & $0.90 \pm 0.28 *$ & $1.33 \pm 0.36$ & $1.44 \pm 0.47$ & $1.32 \pm 0.21$ \\
\hline Kidneys $^{a}$ & $0.37 \pm 0.04$ & $0.20 \pm 0.04^{*}$ & $0.26 \pm 0.05^{*}$ & $0.27 \pm 0.02$ & $0.27 \pm 0.05$ & $0.28 \pm 0.04$ \\
\hline Carcass $^{a}$ & $2.47 \pm 0.29$ & $1.25 \pm 0.13^{*}$ & $1.71 \pm 0.26$ & $1.90 \pm 0.25$ & $1.97 \pm 0.43$ & $2.99 \pm 0.63$ \\
\hline Spleen & $0.22 \pm 0.07$ & $0.16 \pm 0.04$ & $0.23 \pm 0.05$ & $0.21 \pm 0.02$ & $0.12 \pm 0.02$ & $0.15 \pm 0.04$ \\
\hline
\end{tabular}

Values are means \pm SEM; insulin-infused group $n=8$, placebo-infused group $n=5$.

${ }^{a} p<0.05$ refers to individual designated blood flow and resistance values over time by $F$ test for interactions $v s$ placebo-infused group, except for pancreas baseline values were similar between groups.

$* p<0.05 v s$ baseline within each group.

$\dagger p<0.05 v s$ insulin or placebo infusion (time $=48 \mathrm{~h}$ ) within each group.

$\dagger \dagger p<0.02 v s$ placebo-infused group for the same study period by unpaired $t$ test with Bonferroni correction.

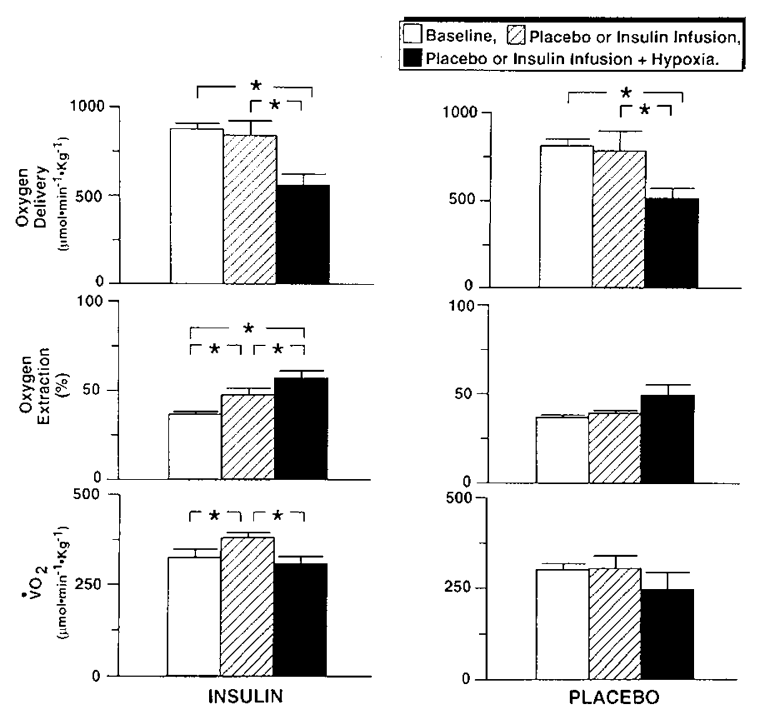

Figure 5. Fetal oxygen delivery, extraction, and uptake $\left(\mathrm{V}_{2}\right)$ in the insulinand placebo-infused groups. Insulin-infused group $n=8$ for oxygen delivery and $n=7$ for extraction and uptake; placebo-infused group $n=5$. Bar and symbol legends as in Figure 1.

and insulin infusion with hypoxia by 38 and $37 \%$, respectively. The glucose extraction ratio increased $(p<0.05)$ from a baseline value of $0.19 \pm 0.04$ during insulin and insulin infusion with hypoxia, by 124 and $137 \%$, respectively. Hypoxia alone was associated with a significant $(p<0.05)$ increase in glucose delivery from a baseline value of $149 \pm 24$ $\mu \mathrm{mol} \cdot \mathrm{min}^{-1} \cdot \mathrm{kg}^{-1}$, by $154 \%$, without changes in extraction.
Glucose uptake demonstrated 50\% nonsignificant increases $(p=0.09)$ during insulin and insulin infusion with hypoxia, and a $130 \%$ nonsignificant increase $(p=0.3)$ during placebo infusion with hypoxia.

\section{DISCUSSION}

The purpose of our study was to investigate the effects of hypoxic hypoxia by acutely limiting oxygen availability upon sympathoadrenal, hemodynamic, and metabolic responses in the hyperinsulinemic ovine fetus. The rationale for doing so is that hyperinsulinism has been suggested to account for many of the morbidities in fetuses of diabetic women (3), who may experience superimposed hypoxia during complications of pregnancy, labor, and delivery. We and others have shown that insulin infusion results in hypoxia and associated changes in hemodynamics and metabolism in the ovine fetus $(6-9,11,12$, 22). In our study, the dose and duration of insulin was selected based upon previous work $(6-12,23)$ and intended to produce sustained hyperinsulinemia for $48 \mathrm{~h}$ to induce hemodynamic changes and accelerate oxygen uptake in the fetus, before exposure to hypoxia. The 15 -fold increase in plasma insulin concentration (Table 2) was sustained during hypoxia and associated with hypoglycemia as previously reported $(9,11$, $12,23)$. It must be emphasized, however, that the effect of hyperinsulinemia in our study differs somewhat from fetuses of diabetic women, who are also exposed to hyperglycemia. In that situation, hyperinsulinemia associated with hyperglycemia may result in increased glycolytic flux. Consequently, the 
HYPOXIA IN HYPERINSULINEMIC FETUSES

Table 4. Oxygen delivery to fetal organs for study groups by study period

\begin{tabular}{|c|c|c|c|c|c|c|}
\hline & \multicolumn{3}{|c|}{ Insulin infusion } & \multicolumn{3}{|c|}{ Placebo infusion } \\
\hline & Baseline & Without hypoxia & With hypoxia & Baseline & Without hypoxia & With hypoxia \\
\hline Time (h) & -1 & 48 & 49 & -1 & 48 & 49 \\
\hline \multicolumn{7}{|c|}{ Oxygen delivery $\left(\mu \mathrm{mol} \cdot \min ^{-1} \cdot 100 \mathrm{~g}^{-1}\right)$} \\
\hline Brain & $360 \pm 33$ & $349 \pm 30$ & $339 \pm 35$ & $411 \pm 30$ & $439 \pm 51$ & $360 \pm 57$ \\
\hline Heart $^{a}$ & $549 \pm 45$ & $1017 \pm 45^{*} \dagger \dagger$ & $1008 \pm 88^{*} \dagger \dagger$ & $547 \pm 23$ & $600 \pm 57$ & $680 \pm 42^{*}$ \\
\hline Adrenal glands ${ }^{a}$ & $533 \pm 85$ & $1285 \pm 171^{*} \dagger \dagger$ & $1171 \pm 203^{*}$ & $795 \pm 102$ & $724 \pm 91$ & $1203 \pm 272$ \\
\hline Liver & $17 \pm 7$ & $34 \pm 8$ & $30 \pm 6$ & $32 \pm 12$ & $44 \pm 9$ & $50 \pm 23$ \\
\hline Gastrointestinal tract & $189 \pm 26$ & $244 \pm 33$ & $124 \pm 22 * \dagger$ & $210 \pm 15$ & $223 \pm 28$ & $108 \pm 28^{*} \dagger$ \\
\hline Pancreas & $143 \pm 20 \dagger \dagger$ & $182 \pm 32$ & $120 \pm 19$ & $220 \pm 18$ & $256 \pm 48$ & $148 \pm 25$ \\
\hline Kidneys & $412 \pm 41$ & $539 \pm 81$ & $314 \pm 70 \dagger$ & $483 \pm 31$ & $534 \pm 69$ & $293 \pm 44 * \dagger$ \\
\hline Carcass & $58 \pm 5$ & $77 \pm 8^{*}$ & $43 \pm 8 \dagger$ & $70 \pm 3$ & $74 \pm 15$ & $30 \pm 5^{*} \dagger$ \\
\hline Spleen & $996 \pm 208$ & $1114 \pm 389$ & $529 \pm 221$ & $620 \pm 62$ & $1154 \pm 157$ & $663 \pm 158$ \\
\hline
\end{tabular}

Values are means \pm SEM; insulin-infused group $n=8$, placebo-infused group $n=5$.

${ }^{a} p<0.05$ refers to designated oxygen delivery values over time by $F$ test for interactions, $v s$ placebo-infused, baseline values were similar between groups.

$* p<0.05$ vs baseline within each group.

$\dagger p<0.05$ vs insulin or placebo infusion (time $=48 \mathrm{~h}$ ) within each group.

$\dagger \dagger p<0.02 v s$ placebo-infused group for the same study period by unpaired $t$ test for interactions.

response to hypoxia would differ from that of hyperinsulinemia alone. Nevertheless, in this and our previous work $(9-12,22)$, we had sought to investigate the specific effects of hyperinsulinemia.

Hypoxic hypoxia was induced by reducing the inspired oxygen of the ewe to simulate adverse late gestation-related hypoxic events. Limited oxygen availability might pose an additional risk to the hemodynamically and metabolically compensated hyperinsulinemic fetus. A comparable degree of hypoxic hypoxia was achieved in the insulin- and placebo-infused groups, as evidenced by similar oxygen content values. During hypoxia umbilical venous and fetal arterial glucose and arterial lactate concentrations increased in the placebo- but not in the insulin-infused fetal sheep (Table 2). This suggests that the effects of insulin superseded that of hypoxia on these substrates. By continually driving glucose utilization (23) in the insulin sensitive tissues, the supraphysiologic insulin concentrations may have counteracted the hypoxia-induced glycolytic effects, which are in part mediated by the catecholamine surge. Therefore, in the hyperinsulinemic fetus, substrate availability for lactate production was limited during hypoxia. Alternatively, hypoxia during hyperinsulinemia may have been associated with less lactate production, because of less tissue hypoxia inasmuch as blood flow to the peripheral tissues (carcass, Table 3) was relatively sustained during hypoxia. In addition, because the placenta is a major site of lactate production, it is possible that this was the primary source of the elevated lactate concentrations during hypoxia in the placeboinfused group. However, because arteriovenous lactate differences were not measured, the source cannot be determined.

The placebo-infused hypoxic group was enrolled to verify that the 48-h infusion and study procedures did not result in changes in the measured variables. The effects of moderate hypoxia in our study were similar to other reports $(13,14,24$, $25)$, as were the effects of insulin infusion upon sympathoadrenal, hemodynamic, and metabolic responses of the fetus (6-12, 22, 26).

The importance of sympathoadrenal stimulation in support of hemodynamic homeostasis in the hyperinsulinemic adult and fetus has been well documented $(9,11,12,15,22,26)$. In an earlier study (11), we showed that hyperinsulinemic hypoglycemia results in increases in both peripheral plasma norepinephrine and epinephrine concentrations. This is consistent with results of hypoxia alone in which peripheral epinephrine and norepinephrine are also increased (17). In our study, peripheral epinephrine and norepinephrine concentrations increased during hyperinsulinemic hypoglycemia, whereas with the level of hypoxia induced in our study, norepinephrine and not epinephrine concentrations increased significantly.

Hypoxia during insulin infusion was associated with significant elevations in catecholamine concentrations. This 4-fold increase was greater than that reported in normal fetuses of similar gestational ages at comparable at levels of hypoxia (17). Because hypoxia and hypoglycemia are both potent physiologic stimuli, the combination of these may have provided a more potent stimulus for the peripheral catecholamine surge than that of hypoxia alone. Higher catecholamine concentrations may be required to support hemodynamic homeostasis during hypoxic hyperinsulinemia than hypoxia alone, because vascular contractions and reactivity to norepinephrine $(27,28)$ are diminished in the presence of insulin. In this regard, the insulin-associated elevations in heart rate and the reductions in mean arterial blood pressure were eliminated by hypoxia. Moreover, the positive correlation between change in blood flow to the heart and epinephrine concentration (Fig. 4) during insulin infusion with hypoxia suggests the role of epinephrine in the preservation of cardiac perfusion.

The insulin-induced increases in the combined ventricular output and fetal body perfusion and decreases in resistance were sustained when oxygen availability was further limited by hypoxic hypoxia (Figs. 2 and 3). The reduction in resistance was in part accounted for by the insulin-induced reduction in fetal arterial blood pressure possibly because of direct insulin vasodilator effects on arteries and veins (27). Nevertheless, although hypoxia eliminated this blood pressure reduction, systemic vascular resistance remained reduced. The prior insulin-induced increased perfusion to the heart was augmented by hypoxia, whereas the increase in blood flow to the gastro- 
intestinal tract, pancreas, perirenal fat, and kidneys remained unchanged. Thus, with the exception of the augmented perfusion to the heart by hypoxia, the regional vascular responses in the insulin-infused fetal sheep were similar to that in the normal state. This suggests that, similar to the situation with anemic hypoxia, the hyperinsulinemic fetus was able to maintain regional perfusion even when exposed to hypoxic hypoxia (22).

The augmented perfusion to the heart during hypoxia in the hyperinsulinemic fetus probably represents a critical stressrelated compensatory response for survival. Under baseline conditions, oxygen extraction of the heart is near maximal. Consequently, during hyperinsulinemia in adult dogs (26), the increase in myocardial oxygen consumption is achieved by increases in perfusion rather than oxygen extraction. Exposure of the hyperinsulinemic fetus to hypoxia presumably accentuated the metabolic demands of the heart, which had already been accelerated by insulin. The 4-fold increase in perfusion represents extremely high levels $\left(650 \mathrm{~mL} \cdot \mathrm{min}^{-1} \cdot 100 \mathrm{~g}^{-1}\right)$ which might not be able to be sustained after longer exposures to hyperinsulinemia and hypoxia.

Hyperinsulinemia accelerated fetal oxygen metabolism (Fig. 5), consistent with previous work (7). This increase in oxygen uptake was based upon an increase in extraction, because delivery had not changed (7). The increased oxygen extraction was further enhanced when oxygen delivery was limited by hypoxia. Nevertheless, increased extraction was not sufficient to offset the decrease in delivery, because the insulin-related accelerated oxygen metabolism was attenuated by hypoxia.

During hyperinsulinemia, oxygen delivery increased to the heart, adrenal glands, and carcass on the basis of increased perfusion, because arterial oxygen content had decreased, suggesting increased regional metabolic demands (29). When hypoxic hypoxia was added, oxygen delivery remained elevated to some regions such as the heart and adrenal glands and was diminished to others including the carcass, gastrointestinal tract, and kidneys. The increase in oxygen delivery to the heart was greater in the hyperinsulinemic fetus than the normal fetus during limited oxygen availability suggesting that demands for oxygen were augmented by the combination of both conditions. Therefore, in the hyperinsulinemic fetus, diminished oxygenation by hypoxic hypoxia reduced the arterial oxygen content compromising oxygen delivery in some regions (carcass, gastrointestinal tract, and kidneys). This suggests that the sustained increases in perfusion were inadequate to meet some regional metabolic demands (29). The decrease in oxygen delivery to these regions was because of decreased oxygen availability without additional increases in perfusion during hypoxia. Although these findings were similar to that observed in the normal fetuses, in that situation, regional oxygen metabolism has not been augmented by insulin (30).

In summary, the hyperinsulinemic ovine fetus exposed to superimposed moderate hypoxic hypoxia demonstrates: $l$ ) an augmented surge in peripheral norepinephrine concentrations above that of hyperinsulinemia alone, 2) sustained insulinrelated increases in the combined ventricular output and fetal body blood flow, 3) augmented blood flow to the heart, 4) an attenuation of the insulin-mediated accelerated systemic oxy- gen uptake, 5) preservation of the elevated oxygen delivery to certain vascular beds (heart and adrenal glands), and 6) diminished oxygen delivery to other regions (carcass, gastrointestinal tract, and kidneys). We conclude that hypoxic hypoxia diminishes the magnitude of the prior insulin-related increase in systemic oxygen uptake in the hyperinsulinemic state. Regional oxygen transport is preserved to vital regions especially the heart by increased perfusion and compromised to other regions, because the increase in perfusion is insufficient to offset the degree of hypoxia. Moreover, although limiting oxygen availability in the hyperinsulinemic state compromises some aspects of the fetal compensatory response to hyperinsulinemia, there are no major qualitative differences in the responses of hyperinsulinemic and normal fetuses to hypoxia.

Acknowledgments. The authors thank Lisa Boyle, Armondo Signore, and Linda Tardoff for their excellent technical assistance

\section{REFERENCES}

1. McFarlane CM, Tsakalakos N 1985 Evidence of hyperinsulinemia and hypoxemia in cord blood of neonates born to mothers with gestational diabetes. South Afr Med J 67:81-84

2. Mimouni F, Miodovnik M, Siddiqi TA, Knouts J, Tsang RC 1988 Perinatal asphyxia in infants of insulin-dependent diabetic mothers. J Pediatr 113:345-353

3. Cowett RM, Schwartz R 1982 The infant of the diabetic mother. Pediatr Clin North Am 29:1213-1231

4. Gutgesell HP, Speer ME, Rosenberg HS 1980 Characterizations of the cardiomyopathy in infants of diabetic mothers. Circulation 61:441-450

5. Young JB, Cohen WR, Rappaport EB, Landsberg L 1979 High plasma norepineph rine concentrations at birth in infants of diabetic mothers. Diabetes 28:697-699

6. Milley JR 1987 Effect of insulin on the distribution of cardiac output in the fetal lamb. Pediatr Res 22:168-172

7. Milley JR, Papacostas JS, Tabata BK 1986 Effect of insulin on uptake of metabolic substrates by the sheep fetus. Am J Physiol 251:E349-E356

8. Milley JR, Rosenberg AA, Phillips AF, Molteni RA, Jones MD, Simmons MS 1984 The effect of insulin on ovine fetal oxygen extraction. Am J Obstet Gynecol 149:673-678

9. Stonestreet BS 1990 Effect of prolonged fetal hyperinsulinemia on plasma catecholamines, circulation and oxygen metabolism in utero. Dev Pharmacol Ther 15:35-44

10. Stonestreet BS, Goldstein M, Oh W, Widness JA 1989 Effect of prolonged hyperinsulinemia on erythropoiesis in fetal sheep. Am J Physiol 257:R1199-R1204

11. Stonestreet BS, Piasecki GJ, Susa JB, Jackson BT 1989 Effects of insulin infusion on plasma catecholamine concentration in fetal sheep. Am J Obstet Gynecol 160:740745

12. Stonestreet BS, Le E, Berard DJ 1993 Circulatory and metabolic effects of $\beta$-adrenergic blockade in the hyperinsulinemic ovine fetus. Am J Physiol 265:H1098-H1106

13. Cohn HE, Sacks EJ, Heymann MA, Rudolph AM. 1974 Cardiovascular responses to hypoxemia and acidemia in fetal lambs. Am J Obstet Gynecol 120:817-824

14. Rurak DW, Richardson BS, Patrick JE, Carmichael L, Homan J 1990 Blood flow and oxygen delivery to fetal organs and tissues during sustained hypoxemia. Am J Physiol 258:R1116-R1122

15. Cohen WR, Piasecki GJ, Cohn HE, Susa JB, Jackson BT 1991 Sympathoadrenal responses during hypoglycemia, hyperinsulinemia and hypoxemia in the ovine fetus. Am J Physiol 261:E95-E102

16. Cohen WR, Piasecki GJ, Cohn HE, Young JB, Jackson BT 1984 Adrenal secretion of catecholamines during hypoxemia in fetal lambs. Endocrinology 114:383-390

17. Cohen WR, Piasecki GJ, Jackson BT 1982 Plasma catecholamines during hypoxemia in fetal lamb. Am J Physiol 243:R520-R522

18. Heymann MA, Payne BD, Hoffman JIE, Rudolph AM 1977 Blood flow measurements with radionuclide-labelled particles. Progr Cardiovasc Dis 20:55-79

19. Bergmeister HU, Bernt E, Schmidt F, Stork H 1974 In: Bergmeyer HU (ed) Methods of Enzymatic Analysis. Academic Press, New York, pp 1196-1201

20. Hales CN, Randle PJ 1963 Immunoassay of insulin with insulin-antibody precipitate. Biochem J 88:137-146

21. Hjemdahl PM, Daleskog M, Kahan T 1979 Determination of plasma catecholamines by high-performance liquid chromatography with electrochemical detection: comparison with a radioenzymatic method. Life Sci 25:131-138

22. Papparella A, Berard DJ, Stonestreet BS 1994 Circulatory and metabolic effects of anemia in the hyperinsulinemic ovine fetus. Am J Physiol 266:H250-H257

23. Hay WW, Meznarick HK 1986 The effect of hyperinsulinemia on glucose utilization and oxidation and on oxygen consumption in the fetal lamb. Q J Exp Physiol 71:689-698 
24. Peeters LH, Sheldon RE, Jones Jr MD, Makowski EL, Meschia G 1974 Blood flow to fetal organs as a function of arterial oxygen content. Am J Obstet Gynecol 135:637-646

25. Rurak DW, Richardson BS, Patrick JE, Carmichael L, Homan J 1990 Oxygen consumption in the fetal lamb during hypoxemia with progressive acidemia. Am J Physiol 258:R1108-R1115

26. Liang C, Doherty JU, Faillace R, Maekawa K, Arnold S, Gravis H, Hood Jr WB 1982 Insulin infusion in conscious dogs. J Clin Invest 69:1321-1336
27. Alexander WD, Oake RJ 1977 The effect of insulin on vascular reactivity to norepinephrine. Diabetes 26:611-614

28. Yagi S, Takata S, Kiyokawa H, Yamamoto M, Noto Y, Ikeda T, Hattori N 1988 Effects of insulin on vasoconstrictive responses to norepinephrine and angiotensin Il in rabbit femoral artery and vein. Diabetes 37:1064-1067

29. Wilkening RB, Molina RD, Battaglia FC, Meschia G 1987 Effect of insulin on glucose/oxygen and lactate/oxygen quotients across hinder of fetal lambs. Biol Neonate $51: 18-23$ 